\title{
INDICE DE MASSA CORPORAL E INDICADORES ANTROPOMÉTRICOS DE ADIPOSIDADE EM IDOSOS RESIDENTES EM ÁREAS RURAIS
}

\author{
Keila Oliveira Diniz ${ }^{1}$ \\ Saulo Vasconcelos Rocha² \\ Clarice Alves Santos ${ }^{3}$
}

resumo

Objetivou-se comparar a concordância de indicadores antropométricos de sobrepeso/obesidade entre idosos residentes em áreas rurais. É um estudo de corte transversal com população de 95 indivíduos com idade igual ou acima de 60 anos. Na avaliação antropométrica, foram utilizadas as variáveis, circunferência da cintura e do braço, a dobra cutânea tricipital e o Índice de Massa Corporal. Na análise dos dados, foram utilizados procedimentos da estatística descritiva e

1 Graduada em Educação Física. Mestranda em Educação Física pela Universidade Federal de Sergipe (UFS). E-mail: keilaodiniz@gmail.com

2 Graduado em Educação Física. Doutorando em Educação Física. Professor assistente da Universidade Estadual do Sudoeste da Bahia (UESB), vinculado ao Departamento de Saúde. E-mail: svrocha@uesb.edu.br

3 Graduada em Educação Física. Doutoranda em Saúde Coletiva. Professora assistente da Universidade Estadual do Sudoeste da Bahia (UESB), vinculada ao Departamento de Saúde. E-mail: claricealvesef@yahoo.com.br 
medidas de associação para variáveis categóricas e a correlação de spearman com p $\leq$ 0.05. A média de idade dos idosos foi de 73,54 \pm 9,43 anos. A prevalência de sobrepeso/obesidade foi maior nas mulheres para todos os indicadores antropométricos. Verificou-se que o IMC teve correlação estatisticamente significante com outros indicadores antropométricos ( $p \leq 0.05$ ) na análise bruta e quando estratificado por sexo. O IMC é um indicador simples e não invasivo que pode ser utilizado na avaliação do estado nutricional de idosos usuários do Programa de Saúde da Família.

palavras-chave

Índice de Massa Corporal. Antropometria. Idoso.

\section{Introdução}

O crescimento da população idosa é um fenômeno observado em países desenvolvidos e em desenvolvimento (BANCO MUNDIAL, 2011). Esse cenário concorre para mudança nos aspectos relacionados à morbimortalidade da população, em que as doenças crônicas não transmissíveis (DCNT), entre as quais estão doenças cardiovasculares, respiratórias e cardiovasculares e cânceres, passam a ser as principais causas de adoecimento/morte entre idosos (SCHMIDT et al., 2011).

Entre os fatores relacionados às DCNT, principalmente às doenças cardiovasculares, encontra-se o excesso de peso e a distribuição da gordura corporal, sobretudo a gordura localizada na região abdominal (MATSUZAWA, 2010). Dessa forma, o estado nutricional é uma condição que deve ser monitorada durante as etapas da vida (MC DONALD et al., 2009).

Os indicadores antropométricos são utilizados para monitorar o estado nutricional em diferentes grupos populacionais, entre os quais o de idosos por estes serem bons preditores de doenças cardiovasculares, diabetes mellitus e do risco coronariano (PITANGA; LESSA, 2006; HAUN et al., 2009). Tais instrumentos apresentam como vantagens a simplicidade e a rapidez na execução, a facilidade de utilização, o baixo custo, e por ser um método pouco invasivo e de grande aplicabilidade em estudos populacionais (MENEZES; MARUCCI, 2005; NASCIMENTO et al., 2011).

O Índice de Massa Corporal (IMC), dentre outros métodos antropométricos, apresenta boas estimativas do estado nutricional, sendo adotado pelos 
sistemas de vigilância nutricional em unidades básicas de saúde (ANJOS et al., 1992) e caracterizando-se como um dos métodos mais utilizados para avaliar sobrepeso e obesidade entre grupos populacionais (SAMPAIO; FIGUEIREDO, 2005). O IMC possui critérios específicos de classificação para idosos (Nutrition Screening Initiative, 2002), e a literatura sugere uma tolerância maior ao avaliar o nível de IMC em idosos em decorrência da diminuição da estatura, da massa corporal magra e da quantidade de água no organismo desta população (CABRERA; JACOB, 2001, SANTOS; SICHIERI, 2005).

Apesar das evidencias quanto à importância dos indicadores antropométricos para a avaliação do estado nutricional e sua relação com agravos à saúde da população (PITANGA; LESSA, 2006; HAUN et al., 2009; MATSUZAWA, 2010), estudos investigando a relação entre indicadores antropométricos (peso, estatura, perímetro e dobras cutâneas) e agravos negativos à saúde da população idosa, principalmente àquelas residentes em áreas rurais, ainda são escassos na literatura. Nesse sentido, o objetivo deste estudo foi comparar a concordância entre indicadores antropométricos de sobrepeso/obesidade em idosos residentes em áreas rurais.

\section{Métodos}

Este é um estudo de corte transversal realizado no município de Jequié, estado da Bahia, Brasil. O município estudado possui área de $3.227 \mathrm{~km}^{2} \mathrm{com}$ população estimada em 151.895 habitantes (IBGE, 2010).

A população do estudo foi composta por 103 idosos residentes na zona rural do município de Jequié com idade maior ou igual a 60 anos. Foi realizado um censo com os idosos cadastrados na Unidade de Saúde da Família do distrito de Itajurú no ano de 2011.

O critério de inclusão para participar do estudo foram indivíduos com 60 ou mais anos de idade de ambos os sexos. Não foram incluídas pessoas com diagnóstico de demência ou outra doença mental, evidente estado de confusão mental e diagnóstico ou relato de surdez, dificuldade em ficar de pé, usuárias de cadeira de rodas e outros tipos de próteses. A população final foi composta por 95 idosos de ambos os sexos.

Precedendo a coleta dos dados, foi conduzido um treinamento com os entrevistadores e um cálculo do erro técnico de medida. A coleta de dados foi iniciada pela entrevista com os idosos e seguida da avaliação antropométrica.

Todas as medidas antropométricas foram realizadas duas vezes pelos pesquisadores e anotadas por um apontador. Os idosos foram pesados e 
medidos conforme procedimentos padronizados segundo Alvarez e Pavan (2007). Com base nessas medidas, foi determinado o índice de massa corporal de fórmula [IMC = massa corporal $(\mathrm{kg}) /$ estatura $\left.^{2}(\mathrm{~m})\right]$. Os pontos de corte do IMC utilizados foram $\leq 22\left(\mathrm{~kg} / \mathrm{m}^{2}\right)$ baixo peso; $>22$ e $<27$ adequado ou eutrófico; $\geq 27$ sobrepeso (LIPSCHITZ, 1994).

A circunferência de cintura (CC) foi mensurada com uma fita antropométrica flexível no ponto médio entre a última costela e a crista ilíaca, sendo considerada a média das duas medidas. A Organização Mundial de Saúde (WHO, 2000) destaca que valores de CC acima de $80 \mathrm{~cm}$ para mulheres e 94 $\mathrm{cm}$ para homens representam risco elevado, e valores acima de $88 \mathrm{~cm}$ para as mulheres e $102 \mathrm{~cm}$ para os homens representam risco muito elevado de doenças cardiovasculares.

A circunferência do braço (CB) foi mensurada com uma fita antropométrica flexível no ponto médio do comprimento entre o processo acromial da escápula e o olecrano. A CB reflete a composição corpórea total sem distinguir tecido adiposo de massa magra. De acordo com Sizer (1996), são considerados desnutridos aqueles idosos com valores abaixo do percentil 5 e obesos aqueles acima de 85.

A Dobra Cutânea de Tríceps (DCT) foi mensurada com uma fita antropométrica flexível no ponto médio do comprimento entre o processo acromial da escápula e o olecrano. Utilizando-se adipômetro do tipo Lange $\AA$, foram considerados desnutridos aqueles idosos com valores abaixo do percentil 5 e obesos aqueles acima de 85 (SIZER, 1996).

Para a análise e interpretação dos resultados, inicialmente foi verificada a normalidade dos dados por meio do teste de Kolmogorov Smirnov. Em seguida, foram feitas a análise descritiva (média, desvio-padrão e percentis), medidas de associação para variáveis categóricas (teste qui-quadrado) e medidas de correlação de spearman com $\mathrm{p} \leq 0.05$. A tabulação dos dados foi realizada no programa EpiData ${ }^{\circledR}$ e os dados analisados no Statistical Package for the Social Sciences SPSS ${ }^{\circledR} 7.0$.

O estudo foi aprovado pelo Comitê de Ética e Pesquisa da Universidade Estadual do Sudoeste da Bahia com o parecer no 195/2010, seguindo princípios éticos estabelecidos na resolução 196/96 de 10 de outubro de 1996 do Conselho Nacional de Saúde (CNS, 1996). 
A média de idade dos idosos foi de 73,54 \pm , 43 anos, sendo que 57,9\% eram do sexo feminino e $42,1 \%$ do sexo masculino. Entre os entrevistados, a maioria tinha renda < que 1 salário mínimo mensal (96,2\%).

As estimativas quanto à média e ao desvio padrão do IMC e da CC encontram-se na tabela 1.

Tabela 1 - Média, Desvio padrão do IMC e da CC entre idosos residentes em áreas rurais, Jequié, Bahia (2011).

\begin{tabular}{l|c|c}
\hline \multicolumn{1}{c|}{ Variáveis } & Média & Desvio padrão \\
\hline Circunferência da Cintura $(\mathrm{cm})$ & 87,09 & 19,05 \\
\hline Índice de Massa Corporal $\left(\mathrm{kg} / \mathrm{m}^{2}\right)$ & 25,58 & 6,09 \\
\hline
\end{tabular}

A distribuição em percentis das medidas de circunferência de braço e dobra cutânea de tríceps estão descritas na tabela 2. De acordo com o percentil, a DCT e CB estão dentro dos padrões considerados normais.

Tabela 2 - Valores médios dos percentis da CB e DCT de tríceps entre idosos residentes em áreas rurais, Jequié, Bahia (2011).

\begin{tabular}{c|c|c}
\hline Variáveis & Percentil & Valores \\
\hline & 5 & 20,27 \\
Circunferência do Braço (cm) & 50 & 25,50 \\
& 85 & 30,43 \\
\hline & 5 & 6,00 \\
Dobra Cutânea de tríceps (mm) & 50 & 16,00 \\
& 85 & 26,00 \\
\hline
\end{tabular}


Observou-se, na tabela 3, uma elevada prevalência de sobrepeso/obesidade nas mulheres e uma prevalência de baixo peso para os homens na variável IMC. $\mathrm{Na}$ DCT, as mulheres estavam acima dos padrões de referência. Com relação à $\mathrm{CC}$, a maioria dos homens e das mulheres não apresentou risco coronariano de acordo com esse indicador.

Tabela 3 - Indicadores antropométricos estratificados por sexo entre idosos residentes em áreas rurais, Jequié, Bahia (2011).

\begin{tabular}{l|c|c|c|c|c}
\hline \multicolumn{1}{|c|}{ IMC (kg/m²) } & \multicolumn{2}{|c|}{ Feminino } & \multicolumn{2}{c|}{ Masculino } & \multirow{2}{*}{ Valor de p } \\
\hline Baixo peso & $\mathbf{n}$ & $\mathbf{n}$ & 15 & 57,7 & 0.003 \\
\hline Normal & 11 & 42,3 & 13 & 50,0 & - \\
\hline Sobrepeso & 13 & 50,0 & 13 & 17,2 & 0,005 \\
\hline
\end{tabular}

DC Triciptal (mm)

\begin{tabular}{l|c|c|c|c|c}
\hline $\begin{array}{l}\text { Dentro dos padrões (entre o } \\
\text { percentil 5 e o percentil 84) }\end{array}$ & 41 & 53,2 & 36 & 46,8 & 0,028 \\
\hline $\begin{array}{l}\text { Acima dos padrões de referência } \\
\text { (abaixo do percentil 5 e acima } \\
\text { do percentil 85) }\end{array}$ & 11 & 91,7 & 1 & 8,3 & \\
\hline
\end{tabular}

$\mathrm{CC}(\mathrm{cm})$

\begin{tabular}{l|c|c|c|c|c}
\hline Não apresenta risco & 48 & 54,5 & 31 & 88,6 & 0,003 \\
\hline $\begin{array}{l}\text { Risco elevado e/ ou Muito } \\
\text { elevado }\end{array}$ & 40 & 45,5 & 4 & 11,4 & \\
\hline
\end{tabular}

$\mathrm{CB}(\mathrm{mm})$

\begin{tabular}{l|c|c|c|c|c}
\hline Desnutrição & 3 & 75,0 & 1 & 25,0 & 0,236 \\
\hline Sobrepeso & 40 & 54,8 & 33 & 45,2 & 0,937 \\
\hline Normal & 10 & 76,9 & 3 & 23,1 & - \\
\hline
\end{tabular}

IMC = Índice de Massa Corporal; DC = Dobra Cutânea; CC = Circunferência da Cintura; $\mathrm{CB}=$ Circunferência do Braço. 
Na tabela 4, encontram-se os valores da correlação entre IMC e dos indicadores antropométricos, e evidenciou-se uma correlação e níveis estatisticamente significantes entre o IMC e os outros indicadores antropométricos. A CC teve forte correlação com o IMC e a DCT e a CB tiveram uma correlação substancial.

Tabela 4 - Correlação entre IMC e indicadores antropométricos entre idosos residentes em áreas rurais, Jequié, Bahia (2011).

\begin{tabular}{l|c|c}
\hline & \multicolumn{2}{|c}{ Valores } \\
\hline Indicadores & $\rho$ & $p$ valor \\
\hline DC Triciptal $(\mathrm{mm})$ & 0,603 & $<0,001$ \\
\hline $\mathrm{CC}(\mathrm{cm})$ & 0,794 & $<0,001$ \\
\hline $\mathrm{CB}(\mathrm{cm})$ & 0,675 & $<0,001$ \\
\hline
\end{tabular}

DC = Dobra Cutânea; CC = Circunferência da Cintura; CB = Circunferência do Braço

Na tabela 5, observou-se que ao analisar a correlação entre o IMC e os outros indicadores antropométricos investigados estratificados por sexo, houve uma sensível diferença na força da correlação, contudo foram mantidos os níveis de significância dentro dos valores aceitáveis $(\mathrm{p} \leq 0.05)$. A correlação para as mulheres na variável antropométrica CC e CB foi forte e substancial para a DCT. Entre os homens, a correlação foi forte para a CC e fraca para DCT e CB.

Tabela 5 - Correlação entre IMC e indicadores antropométricos estratificados por sexo entre idosos residentes em áreas rurais, Jequié, Bahia (2011).

\begin{tabular}{l|c|c|c|c}
\hline \multirow{2}{*}{ Variáveis } & \multicolumn{2}{|c|}{ Feminino } & \multicolumn{2}{c}{ Masculino } \\
\cline { 2 - 5 } & $\rho$ & $p$ valor & $\rho$ & $p$ valor \\
\hline DC Triciptal $(\mathrm{mm})$ & 0,644 & $<0,001$ & 0,348 & 0,038 \\
\hline$C C(\mathrm{~cm})$ & 0,818 & $<0,001$ & 0,775 & $<0,001$ \\
\hline$C B(\mathrm{~cm})$ & 0,744 & $<0,001$ & 0,481 & 0,003 \\
\hline
\end{tabular}

DC = Dobra Cutânea; CC = Circunferência da Cintura; CB = Circunferência do Braço 
A avaliação do estado nutricional, com base nos indicadores IMC e CB, demonstram que a maioria dos indivíduos investigados encontra-se dentro dos escores de normalidade. Comparado com o Inquérito Nacional de Saúde de 1998/1999, o IMC médio observado na população com 65 e mais anos foi de $25,5 \mathrm{~kg} / \mathrm{m}^{2}$. Em estudo com idosas do grupo de convivência da cidade de Aracaju, Sergipe, o IMC médio foi $26,88 \mathrm{~kg} / \mathrm{m}^{2}$, encontrando-se próximo do resultado do presente estudo (SILVA; PETROSKI, 2009).

Os idosos residentes em áreas rurais, na maioria das vezes, continuam a ocupar o seu tempo livre e a desempenhar as tarefas praticadas ao longo da sua vida como a agricultura, a horticultura, dentre outras (NOGUEIRA; SILVA; SANTOS, 2006), o que pode contribuir no controle do peso corporal.

No estudo de Nagahama, Yuyama e Alencar (2003) com mulheres residentes em áreas rurais, os valores obtidos para CB foram semelhantes aos do presente estudo. Os autores supracitados concluem que as moradoras da área urbana apresentaram reserva adiposa maior que o parâmetro de normalidade, indicando menor atividade braçal quando comparados com moradoras da área rural.

Os resultados do presente estudo apontam para uma elevada prevalência de sobrepeso entre os homens e um perfil de normalidade entre as mulheres quando analisado o indicador CB. Com relação ao IMC, foi observada maior prevalência de obesidade entre as mulheres $(82,8 \%)$ em comparação aos homens $(p<0,05)$. Estes resultados corroboram com os encontrados na literatura (GIGANTE et al., 1997; GUEDES; GUEDES, 1998; SABRY et al., 2002).

Um estudo realizado com 218 idosos residentes na área urbana do município de Joinville, Santa Catarina, verificou valores médios de IMC e DCT entre as mulheres significativamente superiores aos dos homens. Estes, por sua vez, apresentavam valores de CC significativamente superiores aos das mulheres ( $\mathrm{p}<0,05)$ (MASTROENI et al., 2010). Rosa et al. (2011) observaram que mulheres usuárias de serviços de pronto atendimento no sul do Brasil apresentavam maiores prevalências de sobrepeso e obesidade quando comparadas com a população geral. Estes resultados estão em conformidade com achados de outros países (GHARAKHANLOU et al., 2012).

$\mathrm{O}$ excesso de peso corporal nas mulheres idosas pode relacionar-se ao acúmulo de gordura visceral e às comorbidades decorrentes do aumento da expectativa de vida (CABRERA; JACOB, 2001). Estudiosos sugerem que as alterações na redistribuição da gordura corporal ocasionada pelo processo de envelhecimento geram o acúmulo aumentado de gordura visceral e intra-abdominal em virtude da diminuição da gordura subcutânea nos membros 
inferiores e superiores (SEIDELL; VISSCHER, 2000; PERISSINOTTO et al., 2002; POU et al., 2009). Isso ocorre de maneira mais acentuada entre mulheres em idades avançadas do que nos homens na mesma idade.

A avaliação da espessura da DC de tríceps revelou um panorama de normalidade para a composição corporal nos idosos. Em contrapartida, uma quantidade maior de mulheres $(91,7 \%)$ encontrava-se acima dos padrões de referência para distribuição da gordura subcutânea tricipital em comparação aos homens $(8,3 \%)$. No entanto, a associação não foi estatisticamente significante ( $p>0.05)$. Fiatarone-Singh (1998) destaca que ocorre uma redistribuição da gordura periférica para regiões centrais do corpo (acúmulo de gordura visceral e intramuscular) no sexo feminino após os 45 anos de idade, e isto pode justificar os achados do presente estudo.

Observou-se uma alta prevalência de obesidade abdominal entre as mulheres investigadas. Matsuzawa (2010) destaca, em seu estudo, que o acúmulo de gordura visceral é considerado o principal fator de risco para doenças cardiovasculares e metabólicas. Um estudo realizado na Coreia do Sul destacou que a obesidade abdominal foi associada a doenças coronarianas e como um importante fator de risco cardiovascular mesmo entre as mulheres com peso corporal normal (KWON, 2009).

Ao avaliar a correlação do IMC com os outros indicadores antropométricos, verificou-se que o IMC apresentou uma correlação a níveis estatisticamente significantes $(\mathrm{p} \leq 0.05)$ tanto na análise bruta como na estratificação por sexo. Um estudo verificando a correlação do IMC indicadores antropométricos de distribuição da gordura corporal em idosos e adultos, de ambos os sexos, atendidos nos ambulatórios do Hospital das Clínicas da Universidade Federal da Bahia na capital de Salvador, observou uma alta correlação entre IMC e CC $(r=0,86 ; p<0,001)$, (SAMPAIO et al., 2005). Uma elevada correlação entre CC e CB com IMC também foi observada por Santos e Sichieri (2005), em que o IMC se associou com a adiposidade corporal independente do envelhecimento.

A correlação do IMC com a dobra cutânea de tríceps na população adulta americana variou de 0,61 a 0,76, apresentando boa correlação entre o IMC e a medida de adiposidade (BEDOGNI, 2001). No estudo de Santos e Sichieri (2005), a correlação foi menor entre o IMC e a dobra triciptal em homens. Zamboni et al. (1998), avaliando indivíduos de 27 a 78 anos, observaram que, tanto nos homens quanto nas mulheres, a correlação do IMC e da CC foi elevada.

Os resultados encontrados sugerem que o IMC é um indicador que apresenta resultados compatíveis com outros indicadores antropométricos na avaliação de idosos residentes em áreas rurais. Além das vantagens já citadas anteriormente, é importante que o IMC seja utilizado no contexto da 
Unidade de Saúde da Família para que possa reforçar/ampliar as iniciativas educacionais no campo da promoção à saúde e de vigilância do estado nutricional dos usuários.

No contexto da atenção primária Gomes et al., (2009) propõe-se a aplicação da antropometria para a detecção de fatores de risco à saúde, sugerindo a utilização de medidas de triagem (IMC e Perímetro de Cintura), avaliadas pelos Agentes Comunitários de Saúde (ACS), e medidas complementares (Dobras cutâneas e outros Perímetros), avaliada por um professor de educação física e/ou nutricionista previamente treinado.

Em contrapartida, o Sistema de Vigilância Alimentar e Nutricional (SISVAN, 2006), preconiza para procedimentos de diagnóstico e acompanhamento do estado nutricional de idosos a utilização do IMC e recomenda um acompanhamento semestral das medidas através da Caderneta do Idoso e/ou no prontuário, permitindo o controle de seu estado nutricional.

Ressalta-se que o número de idosos avaliados no presente estudo revelou o panorama dos idosos cadastrados na USF. O viés de aferição foi reduzido, pois houve treinamento prévio da equipe e cálculo de erro técnico de medida entre os avaliadores.

Conclui-se que os resultados encontrados no presente estudo indicam que o IMC é um bom indicador e tem correlação com outros indicadores antropométricos de adiposidade corporal em idosos. Nesse sentido, os achados do presente estudo podem servir como insumos para políticas e ações em saúde direcionadas à população idosa residente em âmbito rural.

\title{
BODY MASS INDEX AND ADIPOSITY MEASURES IN ELDERLY RESIDENTS IN RURAL AREAS
}

\begin{abstract}
This study aimed to compare the correlation between overweight/ obesity anthropometric indicators among older adults living in rural areas. Cross-sectional study with a 95 individuals population aged above 60 years. Anthropometric variables were used waist circumference, arm circumference, triceps skinfold thickness and body mass index. The exploratory data analysis was performed by descriptive statistics and association measures to categorical variables and Spearman correlation with $p \leq 0.05$. Elderly mean age was 73.54 \pm 9 , 43. Overweight/obesity prevalence was higher in women on all anthropometric indicators. It was observed that BMI had statisti-
\end{abstract}


cally significant correlation with other anthropometric indicators ( $p \leq$

0.05) in both crude analysis as stratified by gender. BMI is a simple

and noninvasive indicator that can be used to elderly users assess nutritional status in the Family Health Program.

keywords

Body Mass Index. Anthropometry. Aged.

\author{
referências
}

ALVAREZ, Bárbara Regina; PAVAN, André Luiz. Alturas e comprimentos. In: PETROSKI, E. L. Antropometria: técnicas e padronizações. 3. ed. Blumenau: Nova Letra, 2007. p. 31-45.

AMERICAN ACADEMY OF FAMILY PHYSICIANS. AMERICAN DIETETIC ASSOCIATION; NATIONAL COUNCIL ON THE AGING. Nutrition screening e intervention resources for healthcare professionals working with older adults. Washington, 2002. Disponivel em: <http://www.eatright.org/cps/rde/xchg/ada/hs.xsl/nutrition_nsi_ENU_HTML.htm>. Acesso em: 26 ago. 2014.

ANJOS, Luiz Antonio dos. Índice de massa corporal (massa corporal.estatura ${ }^{2}$ ) como indicador do estado nutricional de adultos: revisão da literatura. Revista de Saúde Pública, São Paulo, v. 26, n. 6, p. 431-436, dez. 1992.

ANJOS, Luiz Antonio dos et al. Vigilância nutricional em adultos: experiência de uma unidade de saúde atendendo população favelada. Cadernos de Saúde Pública, Rio de Janeiro, v. 8, n. 1, p. 50-56, jan./maio 1992.

BANCO MUNDIAL. Envelhecendo em um Brasil mais velho: implicações do envelhecimento populacional para o crescimento econômico, a redução da pobreza, as finanças públicas e a prestação de serviços. Washington: The World Bank, 2011.

BEDOGNI, Giorgio et al. Is body mass index a measure of adiposity in elderly women? Obesity Research, Silver Spring, v. 9, n. 1, p. 17-20, Jan. 2001.

BRASIL. Ministério da Saúde, Conselho Nacional de Saúde, Resolução n 196/96, de 10 de outubro de 1996.

CABRERA, Marcos A.S; JACOB FILHO, Wilson. Obesidade em idosos: prevalência, distribuição e associação com hábitos e co-morbidades. Arquivos Brasileiros de Endocrinologia \& Metabologia, São Paulo, v. 45, n. 5, p. 494-501, out. 2001.

CAVALCANTI, Cláudio Barnabé dos Santos; CARVALHO, Sineide Cristina Barbosa do Egito; BARROS, Mauro Virgilio Gomes de. Indicadores antropométricos de obesidade abdominal: revisão dos artigos indexados na biblioteca Scielo. Revista Brasileira de Cineantropometria e Desempenho Humano, Florianópolis, v. 11, n. 2, p. 217-225, abr. 2009.

FIATARONE-SINGH, Maria A. Body composition and weight control in older adults. In: Lamb D.R., Murray R. (Ed.). Perspectives in exercise science and sports medicine: exercise, nutrition and weight control. Carmel: Cooper, 1998. p. 243-288.

GHARAKHANLOU, Reza et al. Medidas antropométricas como preditoras de fatores de risco cardiovascular na população urbana do Irã. Arquivos Brasileiros de Cardiologia, São Paulo, v. 98, n. 2, p. 126-135, jan. 2012. 
GIGANTE, Denise P. et al. Prevalência de obesidade em adultos e seus fatores de risco. Revista de Saúde Pública, São Paulo, v. 31, n. 3, p. 236-246, jun. 1997.

GOMES, Marcius de Almeida et al. Ficha Antropométrica no Núcleo de Apoio a Saúde da Família: o que medir e para que medir? Revista Brasileira de Cineantropometria e Desempenho Humano, Florianópolis, v. 11, n. 2, p. 243-253, dez. 2009.

GUEDES, Dartagnan Pinto; GUEDES, Joana Elisabete Ribeiro Pinto. Distribuição de gordura corporal, pressão arterial e níveis de lipídeos-lipoproteínas plasmáticos. Arquivos Brasileiros de Cardiologia, Londrina, v. 70, n. 2, p. 93-98, nov. 1998.

HAUN, Danilo Ramos; PITANGA, Francisco José Gondim; LESSA, Ines. Razão cintura/ estatura comparado a outros indicadores antropométricos de obesidade como preditor de risco coronariano elevado. Revista da Associação Médica Brasileira, São Paulo, v. 55, n. 6, p. 705-711, jul. 2009.

INSTITUTO NACIONAL DE SAÚDE DOUTOR RICARDO JORGE. Inquérito Nacional de Saúde 1998/1999. Lisboa: INSDRJ, 2001.

INSTITUTO BRASILEIRO DE GEOGRAFIA E ESTATÍSTICA. Resolução nº 6 de 3 de novembro de 2010. Disponível em: <http://www.censo2010.ibge.gov.br/dados_divulgados/index.php?uf=29>. Acesso em: 18 dez. 2011.

KWON, Soyang. Association between abdominal obesity and cardiovascular risk factors in normal-weight Korean women. Health Care for Women International, New York, v. 30, n. 5, p. 447-452, May 2009.

LIPSCHITZ, David A. Screening for nutritional status in the elderly. Primary Care, Philadelphia, v. 21, n. 1, p. 55-67, Mar. 1994.

MASTROENI, Marco Fabio et al. Antropometria de idosos residentes no município de Joinville-SC, Brasil. Revista Brasileira de Geriatria e Gerontologia, Rio de Janeiro, v. 13, n. 1, p. 29-40, jan. 2010.

MATSUZAWA, Yuji. Establishment of a concept of visceral fat syndrome and discovery of adiponectin. Proceedings of the Japan Academy. Series B, Physical and Biological Sciences, Tokyo, v. 86, n. 2, p. 131-140, Feb. 2010.

MC DONALD, Margaret et al. Prevalence, awareness, and management of hypertension, dyslipidemia, and diabetes among United States adults aged 65 and older. Journal of gerontology: biological sciences, Baltimore, v. 64, n. 2, p. 256-263, Feb. 2009.

MENEZES, Tarciana Nobre de; MARUCCI, Maria de Fátima Nunes. Antropometria de idosos residentes em instituições geriátricas, Fortaleza, CE. Revista de Saúde Pública, São Paulo, v. 39, n. 2, p. 169-175, abr. 2005.

MORAIS, Eliane Pinheiro; RODRIGUES, Rosalina Aparecida Partezani; GERHARDT, Tatiana Engel. Os idosos mais velhos no meio rural: realidade de vida e saúde de uma população do interior gaúcho. Texto \& Contexto Enfermagem, Florianópolis, v. 17, n. 2, p. 374-383, abr./jun. 2008.

NAGAHAMA, Dionísia; YUYAMA, Lúcia Kiyoko Ozaki; ALENCAR, Fernando Hélio. Perfil antropométrico de mulheres adultas da área urbana e rural no município de Barcelos, AM. Acta Amazonica, Manaus, v. 33, n. 3, p. 423-430, jan. 2003.

NASCIMENTO, Clarissa Matos et al. Estado nutricional e condições de saúde da população idosa brasileira: revisão da literatura. Revista Médica de Minas Gerais, Viçosa, v. 21, n. 2, p. 174-180, ago. 2011.

NOGUEIRA, Maria Assunção Martins; SILVA, Domingos José Lopes da; SANTOS, José Augusto Rodrigues. Actividade física habitual em idosos portugueses rurais e urbanos. Revista Brasileira de Ciência e Movimento, São Paulo, v. 14, n. 4, p. 23-30, ago. 2006.

PERISSINOTTO, Egle et al. Anthropometric measurements in the elderly: Age and gender differences. British Journal of Nutrition, New York, v. 87, n. 2, p. 177-186, Feb. 2002. 
PITANGA, Francisco José Gondim; LESSA, Ines. Razão cintura-estatura como discriminador do risco coronariano de adultos. Revista da Associação Médica Brasileira, São Paulo, v. 52, p. 157-161, maio/jun. 2006.

POU, Karla M. et al. Patterns of abdominal fat distribution. Diabetes Care, Massachusetts, v. 32, n. 3, p. 481-485, Mar. 2009.

ROSA, Maria Inês da et al. Prevalência e fatores associados à obesidade em mulheres usuárias de serviços de pronto-atendimento do Sistema Único de Saúde no sul do Brasil. Ciência \& Saúde Coletiva, Rio de Janeiro, v. 16, n. 5, p. 2559-2566, maio 2011.

SABRY, Maria Olganê Dantas; SAMPAIO, Helena Alves de Carvalho; SILVA, Marcelo Gurgel Carlos da. Hipertensão e obesidade em um grupo populacional no Nordeste do Brasil. Revista de Nutrição, Campinas, v. 15, n. 2 p. 139-147, maio/ago. 2002.

SAMPAIO, Lilian Ramos; FIGUEIREDO, Vanessa de Carvalho. Correlação entre o índice de massa corporal e os indicadores antropométricos de distribuição de gordura corporal em adultos e idosos. Revista de Nutrição, Campinas, v. 18, n. 1, p. 53-61, jan./fev. 2005.

SANTOS, Débora Martins dos; SICHIERI, Rosely. Índice de massa corporal e indicadores antropométricos de adiposidade em idosos. Revista de Saúde Pública, São Paulo, v. 39, n. 2, p. 163-168, abr. 2005.

SCHMIDT, Maria Inês. Chronic non-communicable diseases in Brazil: burden and current challenges. The Lancet, London, v. 377, n. 9781, p. 61-74, June 2011.

SEIDELL, Jaap C.; VISSCHER, Tommy L. S. Body weight and weight change and their health implications for the elderly. European Journal of Clinical Nutrition, Manchester, v. 54, n. 3, p. 33-39, June 2000.

SILVA, Diego Augusto Santos, PETROSKI, Edio Luiz. Associação Entre Diferentes Proposições de Pontos de Corte para Sobrepeso/Obesidade e Pressão Arterial Elevada em Idosas. Revista da Educação Física/UEM, Maringá, v. 20, n. 3, p. 415-422, 3. trim. 2009.

SISTEMA DE VIGILÂNCIA ALIMENTAR E NUTRICIONAL. Estado Nutricional. [2006]. Disponível em: <http://nutricao.saude.gov.br/sisvan.php?conteudo=estado_nutricional>. Acesso em: 7 ago. 2012.

SIZER, Tommas (Ed.). Standards and guidelines for nutritional support of patients in hospitals. Woreestershire: British Association for Parenteral and Enteral Nutrition, 1996.

WORLD HEALTH ORGANIZATION. Obesity: preventing and managing the global epidemic: report of a World Health Organization Consultation. Geneva: World Health Organization, 2000. WHO Obesity Technical Report Series.

ZOMBONI, Mauro et al. Sagittal abdominal diameter as a practical predictor of visceral fat. International Journal of Obesity and Related Metabolic Disorders, Hampshire, v. 22, n. 7, p. 655-660, July 1998. 
